\title{
The olfactory capability of dogs to discriminate between different quantities of food
}

\author{
Shayla M. Jackson ${ }^{1}$. Glynis K. Martin ${ }^{1}$ - William A. Roberts ${ }^{1}$
}

Accepted: 11 January 2021 / Published online: 23 February 2021

(C) The Psychonomic Society, Inc. 2021

\begin{abstract}
A previous study failed to find evidence that dogs could use olfactory cues to discriminate between 1 and 5 hot dog slices presented on a single trial (Horowitz et al., Learning and Motivation, 44, 207-217, 2013). In the experiments reported here, multiple trials were used to test dogs' ability to use olfaction to choose one of two opaque containers under which a larger number of food items was placed. In Experiment 1, dogs chose between 1 and 5 hot dog slices. In Experiments 2 and 3, we examined dogs' ability to discriminate between numbers of hot dog slices that varied in the numerical distance and the ratio between the smaller and larger quantities. Experiment 4 explored olfactory discrimination between quantities of a different food, dog kibble. Experiments 1-3 all showed that dogs used olfactory stimuli to choose the larger number of hot dog slices, but Experiments 2 and 3 revealed no effects of distance or ratio between numerical quantities. In Experiment 4, dogs failed to discriminate between 1 and 5 pieces of dog kibble. Factors that allow dogs to use olfactory cues to discriminate between quantities are discussed.
\end{abstract}

Keywords Olfaction $\cdot$ Dog $\cdot$ Quantity discrimination $\cdot$ Distance effect $\cdot$ Ratio effect $\cdot$ Food type

Dogs have an excellent sense of smell, which has been well documented by researchers throughout the years. Kalmus (1955) showed that dogs can detect the olfactory difference between human twins, and King et al. (1964) showed that dogs can detect undisturbed scents that are well over a month old. Expanding this work, Lo et al. (2019) showed that dogs discriminated between 20 different pairs of essential oil scents and remembered these discriminations for a month or longer. Further evidence shows that dogs can use the information they gather about scents to form aspects of episodic-like memories (Lo \& Roberts, 2019). Recently, Krichbaum et al. (2020) showed that in an odor span task in which dogs were rewarded for choosing new scents, dogs were able to remember 72 different scents within a session, even when the familiar scent had been presented several trials ago, indicating excellent olfactory working memory ability.

Dogs can detect scents that humans cannot because of their greater number of active genes devoted to detecting scents. Both dogs and humans have about 1,000 genes responsible for scent detection (Zozulya et al., 2001), but about $70 \%$ of these

William A. Roberts

roberts@uwo.ca

1 Psychology Department, Western University, London, Ontario N6A $5 \mathrm{C} 2$, Canada genes are deactivated in humans (Rouquier et al., 1998), whereas only about $20 \%$ are deactivated in dogs (Quignon et al., 2005), meaning dogs are able to detect many odors that humans cannot. Polgár et al. (2016) showed that although scent-bred dogs are superior to other breed groups in a scent-detection task, all dogs evaluated were able to detect the scents at an above-chance level at all but the hardest levels of detection, suggesting keen olfactory sensitivity may be found in all dogs, regardless of breed.

Although several researchers have shown that dogs can smell stronger scents better than weaker scents (Cablk et al., 2008; King et al., 1964), little work has been done to examine dogs' ability to smell differences in quantity. In one study, Horowitz et al. (2013) gave dogs a choice between 1 and 5 hot dog slices. Both quantities were hidden inside folded paper plates. Surprisingly, although dogs paid more attention to the larger quantity during their initial investigation, this apparent preference was not reflected in the choice phase - that is, dogs did not show a significant preference for the larger quantity when choosing between the quantities.

Similar work has been done on olfactory discrimination with elephants. Plotnik et al. (2019) gave elephants several choices between a large quantity and a small quantity of sunflower seeds. The researchers used 11 different ratios of quantities that ranged from 4 to $24 \mathrm{~g}$ of seeds. In each trial, both quantities were hidden inside lidded plastic buckets. 
Unlike dogs in the Horowitz et al. (2013) experiment, elephants performed very well on this task. Their discrimination accuracy improved as the small/large $(\mathrm{S} / \mathrm{L})$ ratio declined, showing a Weber's law effect, and improved the greater the distance between the numbers of seeds but showed no change dependent on the overall magnitude of the food presented.

In contrast to the small amount of research on animals' olfactory numerical discrimination ability, there is a large literature on animals' visual numerical discrimination ability. Many of the numerical discrimination effects found with humans are also found in nonhuman animals (Dehaene, Dehaene-Lambertz, \& Cohen, 1998; Nieder, 2019). Two commonly discussed effects are the distance effect and the ratio effect. The distance effect reveals that quantities with a greater distance between them will be easier to discriminate than quantities with a lesser distance between them (e.g., 3:9 is better than 1:3 because the distance between 3 and $9-6$ - is more than the distance between 1 and $3-2$ ). The ratio effect shows that quantities with a smaller $\mathrm{S} / \mathrm{L}$ ratio are easier to discriminate than quantities with a larger $\mathrm{S} / \mathrm{L}$ ratio (e.g., 1:2 is better than $5: 6$ because 0.5 is less than 0.83 ). Both effects have been found in chimpanzees (Beran, 2001) and pigeons (Roberts, 2010) when dealing with visual quantities. These numerosity effects have been seen as indicative of an approximate number system common to human and nonhuman animals (Brannon \& Merritt, 2011), which codes numerical quantities as approximate values on an internal number line. Numbers are scaled logarithmically on this line, giving rise to both distance and ratio effects (Dehaene, 2001; Piazza, 2010; Roberts, 2005).

When presented with a choice between a large quantity and a small quantity that can be seen, dogs reliably choose the larger quantity, even when this visual information is not available at the time of choice. Recent work has shown that dogs' ability to discriminate visual quantities is fairly developed even at 2 months of age (Petrazzini et al., 2020). In adult dogs, visual quantity discrimination appears to be more complex. As found in other animals, adult dogs perform better on quantities that are farther apart (Ward \& Smuts, 2007) and on lower S/L ratio discriminations when dealing with either food (Petrazzini \& Wynne, 2016) or nonfood (Macpherson \& Roberts, 2013) items.

In the present experiments, we investigated dogs' ability to smell differences in quantity of food items. We followed the procedure of Horowitz et al. (2013) of using different numbers of hot dog slices. Because Horowitz et al. found that dogs failed to discriminate between numbers of hot dog slices on a single trial, we tested dogs over repeated trials using hot dog slices. Given Plotnik et al.'s (2019) findings of numerical distance and ratio effects in elephant olfactory discrimination, we also looked for these effects in dog olfactory discrimination. In a final experiment, we investigated dogs' ability to make quantity discriminations based on odor when a different food, dog kibble, was used.

\section{General experimental procedure}

Two gray, plastic containers, that measured $10 \mathrm{~cm} \times 16 \mathrm{~cm}$ with a height of $5.5 \mathrm{~cm}$, were used to hide the location of each quantity of food. Each container had four parallel slits cut into its base that were $2-\mathrm{cm}$ long and allowed air to flow through the base of the container. These containers sat overturned on top of white paper plates that had a diameter of $22.5 \mathrm{~cm}$. The containers were $0.5 \mathrm{~m}$ apart from one another. Food items were placed on the paper plates and covered by the plastic containers. In Experiments 1-3, food items were 2-cm wide $\times 2$-cm long hot dog sections halved into semi cylinders. In Experiment 4, food items were kibble pieces of each dog's own home-fed kibble. All trials began with the dog placed behind a $122-\mathrm{cm} \times 91-\mathrm{cm}$ sheet of cardboard that prevented the dog from seeing the experimenter set up the trial. The handler held the dog behind this sheet of cardboard, and both the dog and the handler were blind to the experimenter's setup of the trial. Once the trial was set up, the blind was removed, and the containers were revealed. The experimenter sat behind the center line of both containers, opposite the dog, with a fixed gaze set between the containers. At this point, the dog was allowed to move forward toward the containers and approach one container first. The dog was allowed to smell the container for $3 \mathrm{~s}$ before being directed to smell the other container for $3 \mathrm{~s}$. For dogs that did not automatically smell the containers upon approach, smelling the container was encouraged by the experimenter tapping the top of the container and asking "What's that?". All dogs sniffed both containers after receiving encouragement. In all trials, if one container was encouraged, the other was also encouraged to prevent bias toward one container over the other. There were no other auditory cues to the location of the hot dog slices. For dogs that tried to flip the containers during the investigation phase, the experimenter placed a hand on each container to hold them steady.

After both containers had been smelled, the handler pulled the dog back $0.5 \mathrm{~m}$ from the containers and centered the dog between the containers, such that the dog was an equal distance from each container. The dog was held in this position for another $3 \mathrm{~s}$ before being released by the handler. At this point, the dog approached one of the containers and removed the food items underneath it. For dogs that had trouble removing the container on their own, the chosen container was lifted, allowing easy access to the food beneath it. The unchosen container and plate were removed by the experimenter. Once the food under the chosen container had been eaten, the handler retrieved the dog, and the experimenter set up the next trial. Trials proceeded in this manner until the session was complete, with an interval of $30 \mathrm{~s}$ separating trials. The quantity of food on each plate was counterbalanced so that each quantity appeared with equal frequency on each plate in a random order. 


\section{Experiment 1}

Similar to the Horowitz et al. (2013) procedure, dogs were offered a choice between hidden quantities of 1 and 5 hot dog slices in order to determine whether they could smell the larger quantity. However, dogs were given multiple trials on this discrimination, unlike the single-trial procedure used by Horowitz et al.

\section{Subjects and procedure}

Ten pet dogs of various breeds were brought to a testing room on the Western University campus and returned home with their owners after testing. Five of the dogs were male, and five of the dogs were female. Breeds included two English Bulldogs, a Labrador Retriever, a Golden Retriever, two Whippets, a Cockapoo, an English Springer Spaniel, a Rough Collie, and a Labrador Retriever/Bernese Mountain Dog cross. The dogs were fasted for several hours prior to testing, and had constant access to water during testing. Care of all the dogs tested in this and the following experiments followed Canadian Council on Animal Care guidelines and was approved by the Western University Animal Care Committee. Details on the demographics of the dogs used in this and the following experiments can be seen in Table 1 .

On each trial, one of the plates had one hot dog slice on it, and the other plate had five hot dog slices on it, both of which were hidden from sight by the overturned container on top of the plate. The quantity on each plate was counterbalanced so that each quantity appeared with equal frequency on each plate in a random order. The experiment was carried out in a single session that lasted for 20 trials.

\section{Results and discussion}

The results are shown for each dog tested in Fig. 1 as the percentage of trials on which the dog chose the larger quantity (5 slices). Nine of the 10 dogs achieved a score above $50 \%$ choices of the larger quantity, and the remaining dog chose at the chance level. Mean choice of the larger quantity was $70.5 \%(S E M=4.62)$ and significantly exceeded chance, $t(9)$ $=4.44, p=.002, d=1.40$.

These results strongly suggest that dogs given multiple trials on a discrimination between 1 and 5 hot dog slices used olfaction to choose the larger quantity over the smaller quantity and contrast with those of Horowitz et al. (2013) based on a single trial of testing. It may be the case that dogs needed repeated trials to associate the reward outcomes of each choice with the odor cues provided by the different numbers of hot dog slices.

\section{Experiment 2}

Having established that dogs choose the larger number of hot dog slices over repeated trials in Experiment 1, our second experiment offered dogs choices between several different hidden quantities of hot dog slices. Given the findings of Plotnik et al. (2019) with elephants, we sought to find out whether dogs also show distance and ratio effects when discriminating between olfactory quantities. The discriminations included 2 and 4 hot dog slices, 4 and 8 hot dog slices and 4 and 6 hot dog slices. Evidence for the distance effect should appear as dogs choosing the larger quantity with more accuracy on the discrimination of 4 and 8 hot dog slices than on the discriminations of 2 and 4 hot dog slices and 4 and 6 hot dog slices, because the distance between 4 and 8 , which is 4 , is larger than the distance between 2 and 4 and 4 and 6 , which is 2. If the Weber's law ratio effect is shown, dogs should choose the larger quantity with more accuracy on the discriminations of 2 and 4 hot dog slices and 4 and 8 hot dog slices than they should on the discrimination of 4 and 6 hot dog slices, because the $\mathrm{S} / \mathrm{L}$ ratio difference of 2 and 4 and 4 and 8 , which is 0.50 , is smaller than the ratio difference of 4 and 6 , which is 0.67 .

\section{Subjects and procedure}

Ten new pet dogs of various breeds were tested. Five of these dogs were brought onto campus for testing and then returned home with their owners, and the other five were tested in their homes by the experimenter. Four of the dogs were male and six of the dogs were female. Breeds included a West Highland Terrier, a German Shepherd/Australian Shepherd cross, a Bichon Frise, a Great Pyrenees/St. Bernard cross, a Great Pyrenees/Maremma Sheepdog/Akbash cross, a Keeshond, an Australian Shepherd, a Jack Russell Terrier/Beagle cross, a Black and Tan Kentucky Coonhound and a Labradoodle. The dogs were fasted for several hours prior to testing and had constant access to water during testing. One dog, a second Bichon Frise, was excluded from the analysis because it lost engagement and refused to complete the trials.

Each of the 10 dogs was led through 24 choice trials by a handler and the experimenter. These choice trials included eight choice trials of each of the three discriminations: 2 and 4 hot dog slices, 4 and 8 hot dog slices, and 4 and 6 hot dog slices. Trials were presented to dogs in a random order within a single session.

\section{Results and discussion}

Figure 2 shows the mean percentage correct choices made on each type of trial. Although dogs scored above chance on each discrimination, the data suggest little difference among conditions. Overall, dogs chose the larger quantity on $63.75 \%$ (SEM $=2.49$ ) of the trials, and this mean significantly exceeded 
Table 1 Demographics of the dogs used in Experiments 1-4

\begin{tabular}{|c|c|c|c|c|c|}
\hline Dog & Age (years) & Breed & Sex & Spay/Neuter Status & Experiment(s) \\
\hline Annabelle & 4 & English Bulldog & $\mathrm{F}$ & $\mathrm{N}$ & 1 \\
\hline Lucy & 8.5 & Labrador Retriever & $\mathrm{F}$ & $\mathrm{Y}$ & 1 \\
\hline Frank & 3.5 & English Bulldog & M & $\mathrm{N}$ & 1 \\
\hline Maia & 5 & Golden Retriever & $\mathrm{F}$ & $\mathrm{Y}$ & 1 \\
\hline Jasper & 4 & Whippet & M & $\mathrm{Y}$ & 1 \\
\hline Finnegan & 3 & Whippet & M & $\mathrm{Y}$ & 1 \\
\hline Bilbo & 2 & Cockapoo & M & $\mathrm{Y}$ & 1 \\
\hline Nutmeg & 5 & English Springer Spaniel & $\mathrm{F}$ & $\mathrm{Y}$ & 1 \\
\hline Cash & 6 & Rough Collie & M & $\mathrm{N}$ & 1 \\
\hline Garnett & .21 & Labrador Retriever/Bernese Mountain & $\mathrm{F}$ & $\mathrm{N}$ & 1 \\
\hline Malina & 14 & West Highland Terrier & $\mathrm{F}$ & $\mathrm{Y}$ & 2 \\
\hline Luna & .25 & German Shepherd/Australian Shepherd & $\mathrm{F}$ & $\mathrm{N}$ & 2 \\
\hline Sophie & 9.5 & Labradoodle & $\mathrm{F}$ & $\mathrm{Y}$ & 2 \\
\hline Jess & 5.5 & Bichon Frise & $\mathrm{F}$ & $\mathrm{Y}$ & 2 \\
\hline Moose & 2.5 & Great Pyrenees/St. Bernard & $\mathrm{F}$ & $\mathrm{Y}$ & 2,4 \\
\hline Razz & 7 & Keeshond & M & Y & 2,4 \\
\hline Akira & 3.5 & Great Pyrenees/Maremma/Akbash & $\mathrm{F}$ & $\mathrm{Y}$ & 2,4 \\
\hline Dallas & 8 & Australian Shepherd & M & Y & 2 \\
\hline Diesel & 10 & Jack Russel Terrier & M & Y & 2 \\
\hline Brody & 8 & Black and Tan Coonhound & M & Y & 2 \\
\hline Blizzard & 7 & Husky & $\mathrm{F}$ & $\mathrm{Y}$ & 3 \\
\hline Indigo & .67 & Alaskan Malamute/Husky/German Shepherd & $\mathrm{F}$ & Y & 3 \\
\hline Athena & 5 & Great Dane & $\mathrm{F}$ & $\mathrm{N}$ & 3 \\
\hline Luke & 1 & $\begin{array}{l}\text { Great Pyrenees/Bouvier des Flandres/ } \\
\text { German Shepherd }\end{array}$ & M & Y & 3 \\
\hline Sophie & .25 & Great Pyrenees/Akbash & $\mathrm{F}$ & $\mathrm{N}$ & 3 \\
\hline Dante & 8 & Labrador Retriever & M & $\mathrm{Y}$ & 3 \\
\hline Tioga & 6 & Labrador Retriever & $\mathrm{F}$ & $\mathrm{Y}$ & 3 \\
\hline Henry & 2.5 & English Pointer & M & $\mathrm{Y}$ & 3 \\
\hline Jorga & 6 & Labradoodle & $\mathrm{F}$ & $\mathrm{Y}$ & 3,4 \\
\hline Jemma & 5 & Labrador Retriever/Rough Collie & $\mathrm{F}$ & $\mathrm{Y}$ & 3,4 \\
\hline Dean & 3.6 & Siberian Husky/Labrador Retriever & M & $\mathrm{Y}$ & 4 \\
\hline Gus & 5 & Shih Tzu & M & $\mathrm{Y}$ & 4 \\
\hline Skyler & 4.5 & Chihuahua & $\mathrm{F}$ & Y & 4 \\
\hline Miley & 12 & Labrador Retriever & $\mathrm{F}$ & $\mathrm{Y}$ & 4 \\
\hline Maddie & 2 & Golden Retriever/Labrador Retriever & $\mathrm{F}$ & $\mathrm{N}$ & 4 \\
\hline
\end{tabular}

chance, $t(9)=5.53, p<.001, \mathrm{~d}=1.75$. An analysis of variance (ANOVA) performed across testing conditions showed no significant effect of type of trial, $F(2,18)=0.31, p=.737$, $\eta_{\mathrm{p}}{ }^{2}=0.03$.

The finding of Experiment 1 was replicated, as dogs again used odor cues to show a significant overall preference for the larger quantity of food. Contrary to the findings of Plotnik et al. (2019) with elephants, dogs did not show superior accuracy when the difference in number of hot dog slices was larger (4 versus 8 ) than when the difference was smaller ( 2 versus 4 and 4 versus 6 ). Also in contrast to the elephant data, dogs did not discriminate better at a lower ratio between quantities ( 2 versus 4 and 4 versus 8 ) than at a higher ratio (4 versus 6 ).

\section{Experiment 3}

In the third experiment, we examined the possibility that dogs did not show significant differences between the conditions tested in Experiment 2 because the differences and ratios used were not sufficiently far apart. To determine whether dogs 


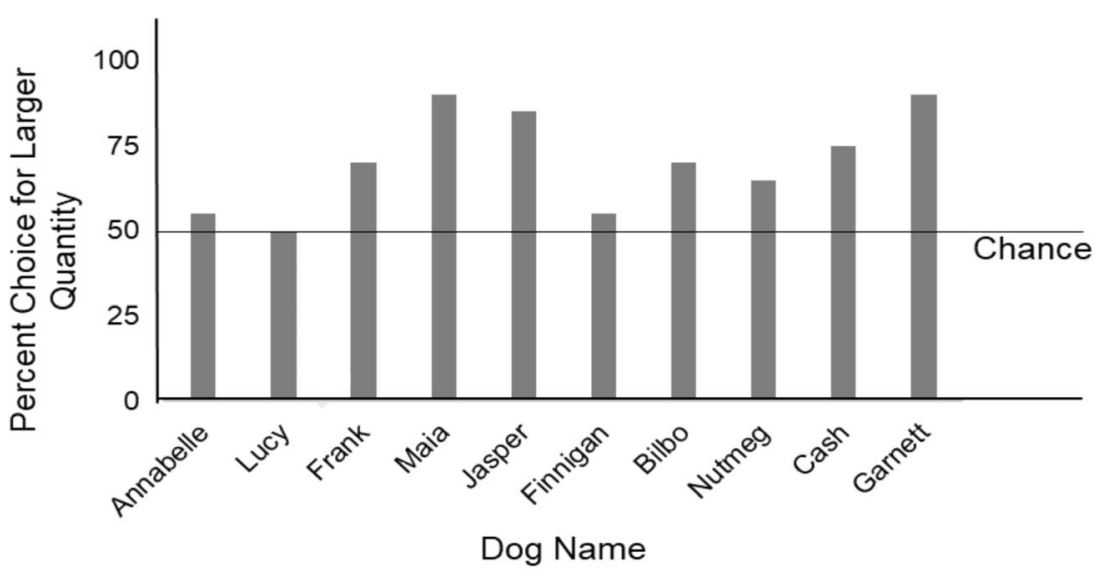

Fig. 1 Percentage choice of the larger quantity when given a choice between one and five hot dog slices. On average, dogs chose the larger quantity more often than they chose the smaller quantity

might show either the distance effect or the ratio effect when discriminating olfactory quantities with wider distance and ratio differences than used in Experiment 2, the discriminations tested in Experiment 3 included 1 and 3 hot dog slices, 3 and 9 hot dog slices, and 8 and 10 hot dog slices. If the distance effect is shown, dogs should choose the larger quantity with more accuracy on the discrimination of 3 and 9 hot dog slices than they should on the discriminations of 1 and 3 hot dog slices and 8 and 10 hot dog slices, because the distance between 3 and 9, which is 6 , is larger than the distance between 1 and 3 and 8 and 10, which is 2. Compared with Experiment 2, the difference between these distances, which is 4, is higher than the difference of 2, used in Experiment 2. Experiment 3 then attempted to increase the saliency of the distance differences to the dogs.

If the ratio effect is shown, the dogs should choose the larger quantity with more accuracy on the discriminations of 1 and 3 hot dog slices and 3 and 9 hot dog slices than they should on the discrimination of 8 and 10 hot dog slices, because the ratio difference of 1 and 3 and 3 and 9, which is 0.33 , is smaller than the ratio difference of 8 and 10 , which is 0.8 .

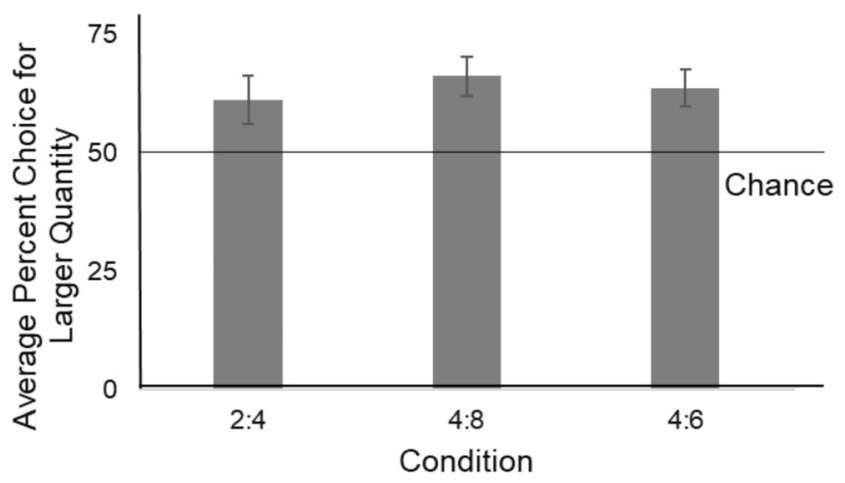

Fig. 2 Percentage choice of the larger quantity at three different discrimination pairs. Dogs did not vary their performance with trial type, but did choose the larger quantity significantly above chance overall. Error bars denote standard error
The difference between these ratios, which is 0.47 , is higher than the difference of 0.17 used between the ratios tested in Experiment 2. Experiment 3 then also attempted to increase the saliency of the ratio differences to the dogs.

\section{Subjects and procedure}

Ten new pet dogs of various breeds were tested. Three of the dogs were male and seven of the dogs were female. Breeds included a Husky, an Alaskan Malamute/Husky/German Shepherd cross, a Great Dane, a Great Pyrenees/Bouvier des Flandres/German Shepherd cross, a Great Pyrenees/Akbash cross, two Labrador Retrievers, an English Pointer, a Labradoodle, and a Labrador Retriever/Rough Collie cross. The dogs were fasted for several hours prior to testing and had constant access to water during testing.

Each of the 10 dogs was tested for one session in its home. During testing, each dog received four choice trials of each of the three discriminations: 1 and 3 hot dog slices, 3 and 9 hot dog slices, and 8 and 10 hot dog slices, totaling 12 choice trials per dog. By increasing the difference between the distance and ratio differences from those used in Experiment 2, the total number of hot dog slices a dog could obtain per trial was increased. To avoid effects of satiation and/or overfeeding, the number of trials per dog was decreased from 24 trials in Experiment 2 to 12 trials in Experiment 3. The experimenter and a handler then led the dogs through 12 trials on which the different conditions were presented in random order, with each number of hot dog slices left-right counterbalanced within each condition.

\section{Results and discussion}

The results are shown in Fig. 3 as the mean choice of the larger quantity for each condition. The overall choice of the larger number of hot dog slices was $57.5 \%(S E M=4.88)$ and this mean was significantly higher than chance, $t(9)=11.80, p<$ 


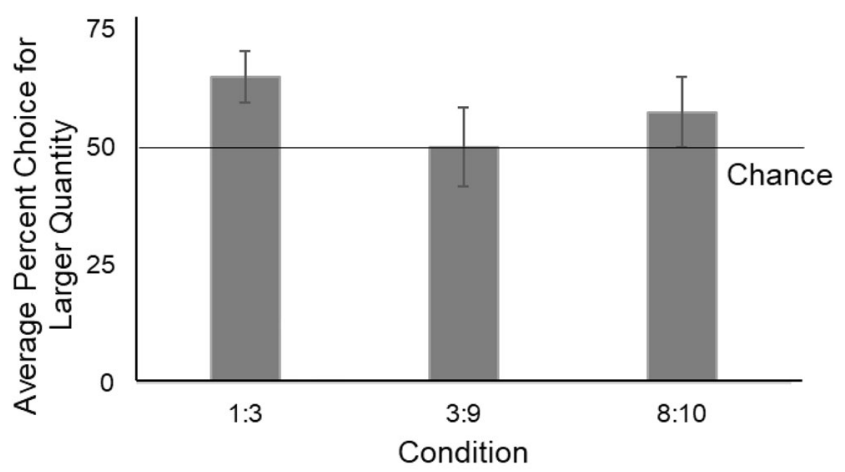

Fig. 3 Average percentage choice of the larger quantity at three different discrimination pairs. Dogs did not vary their performance with trial type, but did choose the larger quantity significantly above chance overall. Error bars denote standard error

$.001, d=3.72$. However, the data failed to offer any suggestion that dogs performed better at a larger distance or lower ratio between numbers of hot dog slices. In fact, dogs chose least accurately on the 3:9 condition with the largest difference and smallest ratio between numbers of hot dog slices. An ANOVA showed no difference among the three conditions tested, $F(2,18)=1.33, p=.290, \eta_{\mathrm{p}}^{2}=0.13$.

These findings confirm those of Experiment 2. Once again, dogs chose the larger quantity significantly above chance. Unlike the elephants tested by Plotnik et al. (2019), however, dogs failed to show that olfactory discrimination between number of food items was controlled by the distance or by the $\mathrm{S} / \mathrm{L}$ ratio between them.

\section{Experiment 4}

Having established that dogs can discriminate between different numbers of hot dog slices using olfaction, we carried out a further experiment to find out whether this ability would extend to a different type of food. In the fourth experiment, dogs were offered a choice between a hidden quantity of 1 kibble piece and a hidden quantity of 5 kibble pieces in order to determine whether they could smell the larger quantity. Because each dog was tested using its own home-fed kibble brand, kibble pieces varied in size and content, but were the same numbers for each dog. We conducted this experiment to see whether dogs' preference for the larger quantity would decrease or remain the same as in the previous experiments when discriminating between quantities of a less preferred and less odorous food item (kibble pieces as compared with hot dog slices).

\section{Subjects and procedure}

Ten pet dogs of various breeds were tested. Five of the dogs were new to this series of experiments, and five of the dogs had been used in one of the previous experiments. All of these dogs were tested in their homes by the experimenter. Three of the dogs were male, and seven of the dogs were female. Breeds included a Keeshond, a Labrador Retriever/Rough Collie cross, a Labradoodle, a Siberian Husky/Labrador Retriever cross, a Great Pyrenees/Maremma Sheepdog/ Akbash cross, a Great Pyrenees/St. Bernard cross, a Shih Tzu, a Chihuahua cross, a Black Labrador Retriever and a Golden Retriever/Labrador Retriever cross. The dogs were fasted for several hours prior to testing and had constant access to water during testing.

Each of the 10 dogs completed an initial food preference trial. The purpose of this initial trial was to find out whether dogs preferred hot dogs over kibble. Preference for hot dogs would indicate that testing in Experiment 4 with kibble involved a less-preferred food reward than that used in Experiments 1-3. In the food preference trial, a dog was shown a hot dog slice in one of the experimenter's open hands and a piece of its kibble in the other open hand. After being shown each open hand, the dog was allowed to choose between the hands containing each type of food, and its preference was recorded.

All test trials were carried out using the same standard olfactory discrimination procedure used in Experiments 1-3. One of the plates had 1 kibble piece on it, and the other plate had 5 kibble pieces on it, and both quantities were hidden from sight by the overturned containers on top of the plates. The quantity on each plate was counterbalanced so that each quantity appeared with equal frequency on each plate in random order. Each dog was tested for a total of 12 trials.

\section{Results and discussion}

In the initial food preference trial, all dogs chose the hot dog slice over the kibble piece, indicating a strong preference for hot dog slices over kibble pieces. On the 12 trials that offered dogs a choice between 1 and 5 pieces of kibble based on olfactory cues, dogs chose the 5 pieces of kibble alternative on $48.3 \%(S E M=5.09)$ of the trials. This percentage did not differ significantly from chance, $t(9)=-0.33, p=.751, d=$ -0.10 .

Clearly, dogs were not able to use olfactory cues to detect the larger number of pieces of kibble. We compared the results of Experiment 4 with kibble to those from Experiment 1 with hot dog slices. In both experiments, dogs chose between 1 and 5 food items using only olfactory cues. The difference between dogs' preference for 5 over 1 hot dog slices (70.5\%) and their preference for 5 over 1 pieces of kibble (48.3\%) was significant by an independent-samples $t$ test, $t(18)=3.22, p=$ $.005, d=1.44$. This result suggests that dogs' ability to detect differences in food quantities depends on the type of food used and the food odors emitted by different types of food. 


\section{General discussion}

In the Horowitz et al. (2013) experiment, a large sample of $\operatorname{dogs}(N=64)$ were tested on a single trial for their ability to detect the difference between 1 and 5 hot dog slices using olfactory cues. Although $61 \%$ of the dogs chose the larger quantity, this value did not differ significantly from chance $(50 \%)$. The work reported here may be seen as an extension of the Horowitz et al. study. In each of our first three experiments, we found that dogs were able to use olfactory cues to choose the larger quantity of food. The analyses reported used data from multiple test trials in each experiment. Our experiments may have met with successful olfactory discrimination, whereas Horowitz et al. did not, either because dogs learned the discrimination over multiple trials or because testing on multiple trials in each experiment yielded a more reliable result. To more precisely compare our results with those of Horowitz et al., we examined performance on Trial 1 for the data from all 30 dogs tested in Experiments 1-3. This analysis revealed that $24 / 30$ or $80 \%$ of the dogs chose the larger quantity on their first trial. This percentage was significantly above the chance level of $50 \%, p<.001$, using a binomial test. There was no evidence of improvement in performance across trials for any of the three experiments, nor was there evidence of any effect of age, sex, or breed, though samples for these characteristics were admittedly underpowered. Unlike the results of Horowitz et al., dogs were able to detect the difference between quantities of hot dog slices using olfactory cues on their first test trial, and this ability did not improve over trials. Our findings prompt an examination of the procedural differences between our experiments and that of Horowitz et al.

Two factors may be of particular importance. First, our experiments used larger hot dog slices $(2-\mathrm{cm}$ wide $\times 2-\mathrm{cm}$ long sections halved into semi cylinders) than those used by Horowitz et al. (2013; $1.25 \mathrm{~cm}$ sections quartered into wedges). Second, whereas in the Horowitz et al. experiment, plates were held in the experimenter's hands and presented to dogs, we allowed dogs to choose which container to approach first. Horowitz et al. (2013) presented quantities sequentially to dogs, whereas we presented them simultaneously in all our experiments. Horowitz et al. noted dogs "gazing" toward their owners and "glancing" toward the experimenter. Thus, it is possible that the presentation style in which quantities were presented by hand caused dogs to search for person cues, whether those cues were present or not. Given that Horowitz et al. (2013) controlled for dogs' use of possible person cues to the correct plate, searching for such cues could have led the dogs to perform closer to chance level. In comparison, the dogs we tested self-directed their investigations, which could have reduced the chance of dogs searching for person cues, leaving them instead to rely on their olfactory abilities. Our experiments also included many more trials compared with only one trial in the Horowitz et al. experiment. Dogs received
12 to 24 trials in a session in our experiments, but we found no evidence that performance improved over these trials.

Experiments 2 and 3 tested for distance and Weber's law ratio effects using olfactory stimuli. The impetus for these experiments was two findings. First, elephants have shown distance and ratio effects using olfactory cues provided by sunflower seeds (Plotnik et al., 2019). Second, dogs have shown distance and ratio effects when discriminating between visual cues provided by food items (Petrazzini \& Wynne, 2016; Ward \& Smuts, 2007) and nonfood items (Macpherson \& Roberts, 2013). In Experiment 2, dogs discriminated between 2 and 4, 4 and 6, and 4 and 8 hot dog slices, and in Experiment 3, dogs discriminated between 1 and 3, 3 and 9, and 8 and 10 hot dog slices. Our prediction from the elephant experiments of Plotnik et al. (2019) and from the dog experiments using visually accessible stimuli was that dogs given only olfactory cues would show a distance effect by showing better performance on 4 versus 8 items than on 2 versus 4 and 4 versus 6 items in Experiment 2 and by showing better performance on 3 versus 9 items than on 1 versus 3 and 8 versus 10 items in Experiment 3. Also, we predicted that dogs would show better performance on lower $\mathrm{S} / \mathrm{L}$ discriminations by scoring higher on 2 versus 4 items and 4 versus 8 items than on 4 versus 6 items in Experiment 2 and would score higher on 1 versus 3 items and 3 versus 9 items than on 8 versus 10 items in Experiment 3. None of these predictions was confirmed. In general, dogs chose the higher quantity significantly above chance, but showed no significant differences among discriminations that varied in distance or ratio. It may be that dogs' olfactory sense is sufficiently good to detect differences in quantity but not differences or ratios between different pairs of quantities. Alternatively, it is possible that with even larger differences in number and ratio these effects might be found.

The findings of Experiment 4 suggest that dogs' ability to detect differences in quantity of food items based on olfaction may be dependent on the type of food used and the extent to which it emits odor cues. After finding that dogs strongly preferred hot dog slices to pieces of kibble, we found no evidence that dogs could detect differences in amount of kibble using olfaction. Given a choice between containers with 1 and 5 pieces of kibble, dogs chose the 5- pieces container over the 1-piece container at no better than chance accuracy. By contrast, all the dogs but one in Experiment 1 chose the 5 hot dog slices container over the 1 hot dog slice container. Although these findings suggest that differences in odor quantity and quality were responsible for differences in dogs' ability to sense differences in number of food items, the control of odor cues was admittedly crude. Research using an olfactometer to measure the emission of odor molecules by different quantities of different foods should be carried out to obtain more precise evidence about differences in odor cues that give rise to a quantity preference.

These findings may have some practical implications. For the pet owner, understanding that dogs do not seem to conform to the distance or ratio effects when responding to 
olfactory or auditory cues, but do seem to understand the more fundamental "larger than" or "smaller than" rule may be helpful in obedience training. Often in dog training, large accomplishments are rewarded with a "jackpot," or an especially large number of treats (Wye, 2010). One way that dogs could know they are, or will be, receiving more treats than usual is through the olfactory cues of smelling a quantity of treats in their handler's hand. Our research suggests that dogs might understand an especially large number of treats as simply more treats than usual and not perform better for certain amounts of jackpot over others because neither the distance nor the ratio effect was shown. That is, a dog should be just as good at discriminating 4 treats from the usual 2 as they are at discriminating 8 treats from the usual 2. Olfactory cues could be important for motivating dogs with jackpots, both because some dogs may be too excited when receiving treats to perceive any form of visual information about them and because cues are responded to quicker and with more accuracy when presented in several sensory modalities. In fact, many species may even be adapted to respond better to these multimodal, rather than unimodal, cues (Rowe, 1999).

Acknowledgements Support for this research was provided by a Discovery Grant from the Canadian Natural Sciences and Engineering Research Council to W. A. Roberts.

\section{References}

Beran, M. J. (2001). Summation and numerousness judgments of sequentially presented sets of items by chimpanzees (Pan troglodytes). Journal of Comparative Psychology, 115, 181-191. https://doi. org/10.1037/0735-7036.115.2.181

Brannon, E. M., \& Merritt, D. J. (2011). Evolutionary foundations of the approximate number system. In S. Dehaene \& E. Brannon (Eds.), Space, time and number in the brain: Searching for the foundations of mathematical thought (pp. 207-224). Elsevier. https://doi.org/10. 1016/B978-0-12-385948-8.00014-1

Cablk, M. E., Sagebiel, J. C., Heaton, J. S., \& Valentin, C. (2008). Olfaction-based detection distance: A quantitative analysis of how far away dogs recognize tortoise odor and follow it to source. Sensors, 8, 2208-2222. https://doi.org/10.3390/s8042208

Dehaene, S. (2001). Subtracting pigeons: Logarithmic or linear? Psychological Science, 12, 244-246. https://doi.org/10.1111/14679280.00343

Dehaene, S., Dehaene-Lambertz, G., \& Cohen, L. (1998). Abstract representations of numbers in the animal and human brain. Trends in Neurosciences, 21, 355-361. https://doi.org/10.1016/SO1662236(98)01263-6

Horowitz, A., Hecht, J., \& Dedrick, A. (2013). Smelling more or less: Investigating the olfactory experience of the domestic dog. Learning and Motivation, 44, 207-217. https://doi.org/10.1016/j.lmot.2013. 02.002
Kalmus, H. (1955). The discrimination by the nose of the dog of individual human odours and in particular of the odours of twins. The British Journal of Animal Behaviour, 3, 25-31. https://doi.org/10. 1016/S0950-5601(55)80072-X

King, J. E., Becker, R. F., \& Markee, J. E. (1964). Studies on olfactory discrimination in dogs: (3) Ability to detect human odour trace. Animal Behaviour, 12, 311-315. https://doi.org/10.1016/00033472(64)90017-X

Krichbaum, S., Rogers, B., Cox, E., Waggoner, L. P., \& Katz, J. S. (2020). Odor span task in dogs (Canis familiaris). Animal Cognition, 23, 571-580. https://doi.org/10.1007/s10071-02001362-7

Lo, K. H., Macpherson, K., MacDonald, H., \& Roberts, W. A. (2019). A comparative study of memory for olfactory discriminations: Dogs (Canis familiaris), rats (Rattus norvegicus), and humans (Homo sapiens). Journal of Comparative Psychology, 134, 170-179. https://doi.org/10.1037/com0000205

Lo, K. H., \& Roberts, W. A. (2019). Dogs (Canis familiaris) use odor cues to show episodic-like memory for what, where, and when. Journal of Comparative Psychology, 133, 428-441. https://doi. org/10.1037/com0000174

Macpherson, K., \& Roberts, W. A. (2013). Can dogs count? Learning and Motivation, 44, 241-251. https://doi.org/10.1016/j.lmot.2013. 04.002

Nieder, A. (2019). A brain for numbers: The biology of the number instinct. MIT Press.

Petrazzini, M. E. M., Mantese, F., \& Prato-Previde, E. (2020). Food quantity discrimination in puppies (Canis lupus familiaris). Animal Cognition, 23, 703-710. https://doi.org/10.1007/s10071-02001378-z

Petrazzini, M. E. M., \& Wynne, C. D. L. (2016). What counts for dogs (Canis lupus familiaris) in a quantity discrimination task? Behavioural Processes, 122, 90-97. https://doi.org/10.1016/j. beproc.2015.11.013

Piazza, M. (2010). Neurocognitive start-up tools for symbolic number representations. Trends in Cognitive Sciences, 14, 542-551. https://doi.org/10.1016/j.tics.2010.09.008

Plotnik, J. M., Brubaker, D. L., Dale, R., Tiller, L. N., Mumby, H. S., \& Clayton, N. S. (2019). Elephants have a nose for quantity. Proceedings of the National Academy of Sciences of the United States of America, 116, 12566-12571. https://doi.org/10.1073/ pnas. 1818284116

Polgár, Z., Kinnunen, M., Újváry, D., Miklósi, Á., \& Gácsi, M. (2016). A test of canine olfactory capacity: Comparing various dog breeds and wolves in a natural detection task. PLOS ONE, 11, Article e0154087. https://doi.org/10.1371/journal.pone.0154087

Quignon, P., Giraud, M., Rimbault, M., Lavigne, P., Tacher, S., Morin, E., Retout, E., Valin, A., Lindblad-Toh, K., Nicolas, J., \& Galibert, F. (2005). The dog and rat olfactory receptor repertoires. Genome Biology, 6, R83. https://doi.org/10.1186/gb-2005-6-10-r83

Roberts, W. A. (2005). How do pigeons represent numbers?: Studies of number scale bisection. Behavioural Processes, 69(1), 33-43. https://doi.org/10.1016/j.beproc.2005.01.005

Roberts, W. A. (2010). Distance and magnitude effects in sequential number discrimination by pigeons. Journal of Experimental Psychology: Animal Behavior Processes, 36, 206-216. https://doi. org/10.1037/a0017226

Rouquier, S., Taviaux, S., Trask, B. J., Brand-Arpon, V., van den Engh, G., Demaille, J., \& Giorgi, D. (1998). Distribution of olfactory receptor genes in the human genome. Nature Genetics, 18, 243-250. https://doi.org/10.1038/ng0398-243 
Rowe, C. (1999). Receiver psychology and the evolution of multicomponent signals. Animal Behaviour, 58, 921-931. https://doi.org/10. 1006/anbe.1999.1242

Ward, C., \& Smuts, B. B. (2007). Quantity-based judgments in the domestic dog (Canis lupus familiaris). Animal Cognition, 10, 71-80. https://doi.org/10.1007/s10071-006-0042-7

Wye, M. V. (2010). U.S. Publication Number: US 2010/0095896 A1. U.S. Patent and Trademark Office.
Zozulya, S., Echeverri, F., \& Nguyen, T. (2001). The human olfactory receptor repertoire. Genome Biology, 2, Article research0018.1. https://doi.org/10.1186/gb-2001-2-6-research0018

Publisher's note Springer Nature remains neutral with regard to jurisdictional claims in published maps and institutional affiliations. 